\title{
Effectiveness evaluation of two volumizing hyaluronic acid dermal fillers in a controlled, randomized, double-blind, split-face clinical study
}

This article was published in the following Dove Press journal:

Clinical, Cosmetic and Investigational Dermatology

29 June 2017

Number of times this article has been viewed

\author{
Martina Kerscher \\ Karla Agsten ${ }^{2}$ \\ Maria Kravtsov ${ }^{3}$ \\ Welf Prager ${ }^{4}$ \\ 'Department of Cosmetic Science, \\ University of Hamburg, ${ }^{2} \mathrm{SCl}$ derm \\ GmbH, Hamburg, Germany; ${ }^{3}$ Anteis \\ S.A., Geneva, Switzerland; ${ }^{4}$ Prager \& \\ Partner Dermatologische Praxis, \\ Hamburg, Germany
}

Background: Enhancement of the midface can be achieved with volumizing hyaluronic acid (HA) fillers.

Objective: The objective of this study was to compare the safety and effectiveness of Cohesive Polydensified Matrix ${ }^{\circledR} 26 \mathrm{mg} / \mathrm{mL}$ HA gel (CPM-26) and Vycross ${ }^{\circledR} 20 \mathrm{mg} / \mathrm{ml} \mathrm{HA}$ gel (VYC-20) in a controlled, randomized, evaluator-blind, split-face clinical study.

Patients and methods: Subjects with moderate-to-severe malar volume loss on the Merz Aesthetics Scale (MAS) received CPM-26 on one side and VYC-20 on the contralateral side of the face. Effectiveness assessments were performed by blinded evaluators including photographic and live MAS ratings and live Global Aesthetic Improvement Scale (GAIS) ratings. Calculations of anatomical volume variations at month 3 (M3), month 6 (M6), month 12 (M12) and month 18 (M18) were also performed.

Results: Non-inferiority of CPM-26 versus VYC-20 was demonstrated at M3 (primary end point) based on MAS. GAIS rating showed that significantly more subjects had better improvement with CPM-26 than with VYC-20 at month 1, M3, M12 and M18 ( $p=0.0032, p=0.0074$, $p=0.0384$ and $p=0.0110$, respectively). Standardized evaluation of volume variations from baseline to M3, M12 and M18 showed that CPM-26 created more volume augmentation at all time points, and the difference was significant at $\mathrm{M} 3$.

Conclusion: CPM-26 was non-inferior to VYC-20 based on MAS ratings at M3 and demonstrated a favorable safety and effectiveness profile for midfacial volume enhancement with results lasting up to $\mathrm{M} 18$.

Keywords: cohesive polydensified matrix, hyaluronic acid fillers, Belotero ${ }^{\circledR}$ Volume, Modélis ${ }^{\circledR}$ SHAPE, Juvéderm ${ }^{\circledR}$ VOLUMA ${ }^{\circledR}$, volumizing

\section{Introduction}

Midfacial volume loss associated with aging has created a lot of interest in the esthetics domain in recent years. The associated morphophysiological changes described in the literature are cranial bone remodeling, facial sagging due to gravity and loss of soft-tissue fullness in certain areas with hypertrophy of fat in others. ${ }^{1}$ According to expert consensus, volumetric restoration of the midface is the first essential step in global rejuvenation, as it has the most significant impact on the malar contour and the surrounding areas. ${ }^{2-4}$

Currently available hyaluronic acid (HA) volumizing dermal fillers have been specifically developed to provide robust support to the soft tissues from the inside. When injected in the upper cheeks, they accentuate the projection of the zygomaticomalar
Correspondence: Welf Prager

Prager \& Partner Dermatologische

Praxis, Hemmingstedter Weg 168, 22609

Hamburg, Germany

Tel +494081991991

$\mathrm{Fax}+494081991992$

Email info@derma-hamburg.de 
eminence and thus allow restoration of the convex heartshaped face associated with youth and attractiveness. ${ }^{5-8}$

CPM-26 (Modélis ${ }^{\circledR}$ Shape; Anteis S.A., Geneva, Switzerland - now commercialized as Belotero ${ }^{\circledR}$ Volume; Merz Pharmaceuticals GmbH, Frankfurt am Main, Germany) is a Conformité Européene (CE)-marked $26 \mathrm{mg} / \mathrm{mL}$ HA gel manufactured with the patented Cohesive Polydensified Matrix $^{\circledR}\left(\mathrm{CPM}^{\circledR}\right)$ technology. VYC-20 (Juvéderm ${ }^{\circledR}$ Voluma $^{\circledR}$; Allergan Inc., Pringy, France) is a CE-marked and US Food and Drug Administration-approved $20 \mathrm{mg} / \mathrm{mL}$ HA gel manufactured with the patented Vycross ${ }^{\circledR}$ technology.

Safety and performance of CPM-26 $6^{9}$ and VYC-20 $0^{10-13}$ have been individually demonstrated in different publications. The aim of the current study was to compare safety and effectiveness of CPM-26 and VYC-20.

\section{Patients and methods}

\section{Study design}

This randomized split-face study was performed in compliance with ISO14155:2011 and the principles of the Declaration of Helsinki and approved by the ethics committee of the Medical Council of Hamburg, Germany. All patients provided written informed consent prior to any study-related procedures and gave permission for their photographs to be used.

Subjects of both genders between 18 and 65 years of age desiring a volume enhancement in their upper cheeks were recruited from June to September 2013. The main inclusion criteria were bilateral, symmetrical, moderate-tosevere sunken upper cheeks (grades 2 and 3) on the upper cheek fullness Merz Aesthetics Scale (MAS); Fitzpatrick skin type I-IV and realistic treatment expectations. Subjects were excluded in case of concomitant facial procedures such as dermal fillers, photorejuvenation, chemical peeling and dermabrasion during the whole study period. Botulinum toxin injections in the glabella or forehead were not allowed in the preceding 4 weeks and 3 months after the study injections.

All study injections were performed by the same nonblinded investigator, who took maximum care to keep subjects unaware of product allocation, by hiding the boxes and not announcing which product was injected on which side. All study live assessments at baseline and the subsequent follow-up visits were performed by the site-blinded evaluator. Photographic assessments were performed by another independent blinded evaluator. All other study team members, including the sponsor and subcontractors, were also blinded.

\section{Treatment}

Commercially available products were provided to the nonblinded investigator. At day 0 (D0), subjects were randomized to receive a single injection of CPM-26 in one upper cheek and VYC-20 in the opposite cheek. Overcorrection was not allowed, and injection volume was limited to $2 \mathrm{~mL}$. The use of needles/cannulae, injection depth and technique were at the discretion of the investigator, but were required to be identical for both sides of the face. The products were without lidocaine, and the use of anesthetics was not permitted.

Touch-ups were not allowed; however, an exception was introduced for subjects who complained about asymmetrical cheeks after the month 3 (M3) visit. Upon request, these subjects could attend an additional evaluation visit between M3 and month 12 (M12), during which the site-blinded evaluator confirmed the asymmetry if there was at least a 1-grade difference between the cheeks. A touch-up was offered on the under-corrected side with the same product that was initially implanted.

In this study, optimal correction was defined as volumetric enhancement in the midface achieved at M3. The longevity of the effect was evaluated at the month 6 (M6), M12 and month 18 (M18) visits.

\section{Clinical assessments}

Clinical assessments were performed by the site-blinded evaluator at screening (SCR), D0 posttreatment, M1, M3, M6, M12 and M18 visits. Baseline data were recorded at the SCR visit. At each visit, two-dimensional (2D) photographs were taken using a stereovision digital camera (LifeViz ${ }^{\mathrm{TM}}$; QuantifiCare S.A., Sophia Antipolis, France) at $60 \mathrm{~cm}$ distance. Standardized indirect light, aperture, speed and distance of the camera were controlled. Subjects were asked to tie back their hair, remove jewelry and pose with a relaxed neutral face expression.

\section{Primary end point}

Photographic assessment of upper cheek fullness was performed by the independent blinded evaluator on the validated MAS (Figure 1), ranging from full upper cheeks (grade 0) to very severely sunken upper cheeks (grade 4). ${ }^{14}$ Split-face printed subject photographs from SCR and M3 visits were presented in a random order of subjects and visits and were analyzed in a single session. The evaluator was blinded to the identity of subjects, chronology of the visits, product allocation and their quantities. Left-side photographs were mirrored to appear as the right side, so as to further avoid bias. Each side of the face was scored separately. 


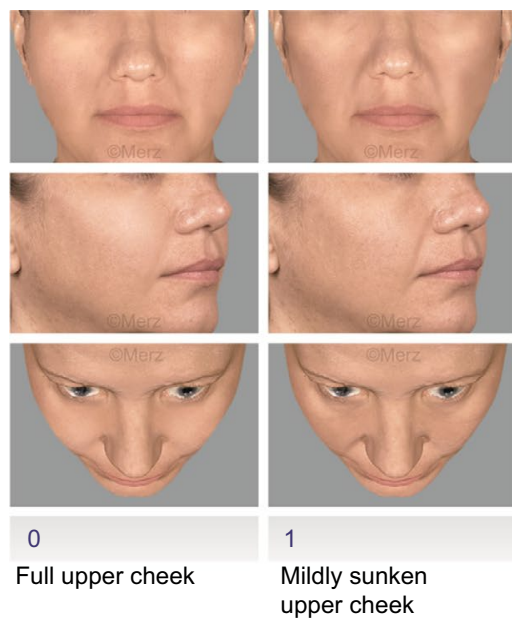

upper cheek

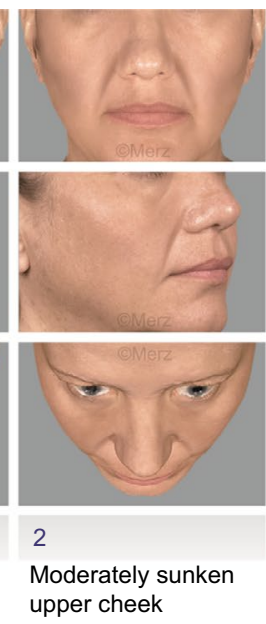

upper cheek
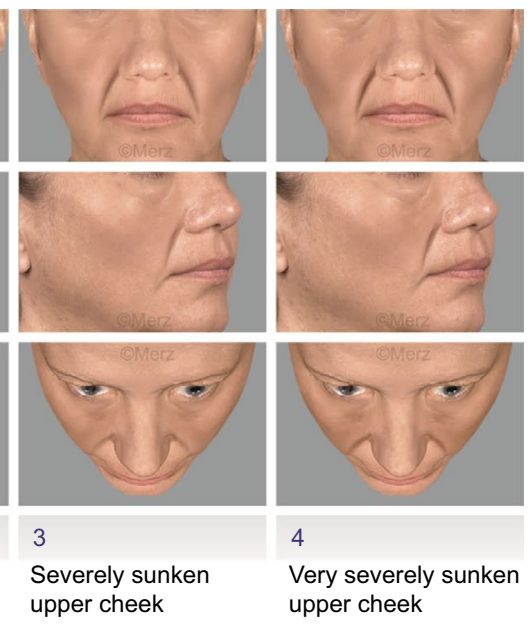

Scâles

Figure I Upper cheek fullness MAS.

Note: Reproduced with permission from Merz Pharmaceuticals GmbH. Abbreviation: MAS, Merz Aesthetics Scale.

\section{Secondary end points}

Live assessment of upper cheek fullness was performed by a specifically trained site-blinded evaluator using the MAS. ${ }^{14}$ Each side of the face was scored separately.

Live assessment was also performed on the 5-point Global Aesthetic Improvement Scale (GAIS), ranging from "very much improved" (grade 3 ) to "worse" (grade -1$).{ }^{15}$ The site-blinded evaluator compared the subjects live with their baseline photographs displayed on the computer screen.

An exploratory assessment of volume variations from SCR to M3, M12 and M18 visits was performed on a subgroup of the 30 first consecutively enrolled subjects without major deviations in the established photographic procedure. Digital photographs were reconstructed in three dimensions (3D) with LifeViz ${ }^{\mathrm{TM}}$ software. Volume changes were measured through an accurate automatic matching of pre- and postinjection images based on anatomical landmarks, such as face oval and the T-zone. Once both surfaces were perfectly matched, anatomical volume variations were calculated in cubic centimeter within the region of interest. The methodology was validated internally by the provider.

\section{Statistics}

The randomization list was generated by an independent statistician using RandList 1.2 software. A sample size of 38 subjects was calculated to achieve a power of $\sim 80 \%$ at a $5 \%$ significance level based on a one-sided equivalence test of correlated proportions. The standard rate and the treatment rate were assumed to be 0.90 , the disagreement rate was $10 \%$ and the non-inferiority margin was set at 0.15 . Assuming a dropout rate of $20 \%$, it was planned to enroll 46 subjects. A total of 50 subjects were screened for the study, and 46 subjects met the study inclusion criteria and were enrolled. One subject was withdrawn for a protocol violation (previous treatment with a permanent filler), and in one patient, treatments were interchanged by mistake, and therefore, 44 patients were considered for the efficacy evaluation.

The primary objective of this study was to demonstrate non-inferiority of CPM-26 compared to VYC-20 in terms of performance in malar volume enhancement. Responders were defined as subjects achieving at least a 1-grade improvement from baseline to M3 on MAS. A one-sided 95\%-adjusted Wald confidence interval for dependent samples was calculated for the difference of responder rates (CPM-26 minus VYC-20). Non-inferiority was concluded if the lower limit of the confidence interval was above the predefined margin of -0.15 . This analysis of the primary efficacy parameter based on the full analysis set (FAS), defined as all subjects who received the treatment and whose primary end point at M3 was available, was regarded as confirmatory analysis. All other efficacy analyses were regarded as exploratory.

The GAIS score and anatomical volume variations were compared between the two treatments at all visits using twosided Wilcoxon signed-rank tests.

All results have been analyzed descriptively on the FAS. Regarding MAS rating and anatomical volume variations, 
data from the asymmetrical subjects referring to the reinjected side were considered as missing. Data from the contralateral side were kept in the analysis. Regarding GAIS, missing values were replaced with the most severe rating that may theoretically have occurred $(-1=$ worse $)$.

\section{Results}

Out of 50 screened subjects, a total of 46 were enrolled in the study. The majority of subjects $(n=44,95.7 \%)$ were women. The mean age was 50.3 years (range: $38-66$ years). The majority of subjects were Caucasian $(n=45)$, except one who was of Hispanic ethnicity. Subjects presented with Fitzpatrick skin types II (23.9\%), III (69.6\%) and IV (6.5\%).

A total of 45 subjects were randomized to receive a single $2 \mathrm{~mL}$ injection of CPM-26 on one side and VYC-20 on the contralateral side of the face. Of the 45 subjects, one had the treatments interchanged by mistake, and the subject was analyzed as treated and not as randomized.

Four subjects complained about asymmetry in their cheeks between the M3 and M6 follow-up visits (Figure 2). During the additional evaluation visits, the site-blinded evaluator confirmed a difference of at least 1 grade on MAS between the left and right upper cheeks for the four subjects. In all four cases, the side previously injected with VYC-20 appeared undercorrected and was reinjected with the same product. An average of $1.45 \mathrm{~mL}$ of VYC-20 was administered (range: $0.8-2 \mathrm{~mL}$ ).

\section{Primary end point}

The responder rate on the side treated with CPM-26 (56\%) was slightly higher than the responder rate on the side treated with VYC-20 (47\%) at M3. Statistical analysis confirmed that the responder rate of CPM-26 was significantly non-inferior to VYC-20 (difference of responder rate: 0.09 , one-sided $95 \%$ CI: $[-0.07,1])$.

\section{Secondary end points}

Based on MAS live assessment at SCR, all subjects had bilateral grade 2 or 3 . At M3, $100 \%(n=45)$ of MAS scores improved on the CPM-26 side and $98 \%(n=44)$ on the VYC-20 side. At this optimal correction time point, the responder rate of CPM-26 was significantly non-inferior to the responder rate of $\mathrm{VYC}-20$ (difference of responder rate: 0.02 , one-sided $95 \% \mathrm{CI}:[-0.04,1])$, and the primary objective was confirmed.

Long-lasting results were supported by improvement reported at M12 and M18. A total of $81 \%(n=34)$ and $54 \%$ $(n=22)$ of scores were still improved by at least 1 grade on the CPM-26 side at M12 and M18, respectively. A total of $68 \%(n=26)$ and $51 \%(n=19)$ of scores were still improved by at least 1 grade on the VYC-20 side at M12 and M18, respectively.

GAIS ratings by the site-blinded evaluator during live assessment demonstrated overall satisfaction with both treatments. As presented in Figure 3, both products demonstrated best grades in terms of esthetic improvement at M1, with $71 \%(n=32)$ and $51 \%(n=23)$ of subjects found in grade " 3 - very much improved" for CPM-26 and VYC20 , respectively. Most subjects had grades " 2 " and " 3 " on both sides from D0 to M6 (Figure 3A-D), and a shift toward lower scores was visible at M12 and M18 (Figure 3E and F). Only a few subjects returned to baseline scores at M12 (one on CPM-26 side and two on VYC-20 side). At M18, 78\% $(n=35)$ of subjects showed an improvement (subjects with grades 1, 2 and 3) with CPM-26. Likewise, on the VYC-20 side, 65\% $(n=29)$ of subjects showed an improvement at M18.

At each time point, more subjects had grade "3 very much improved" on the CPM-26 side than on the VYC-20 side. Significantly more subjects showed best grades in terms of esthetic improvement on the CPM-26

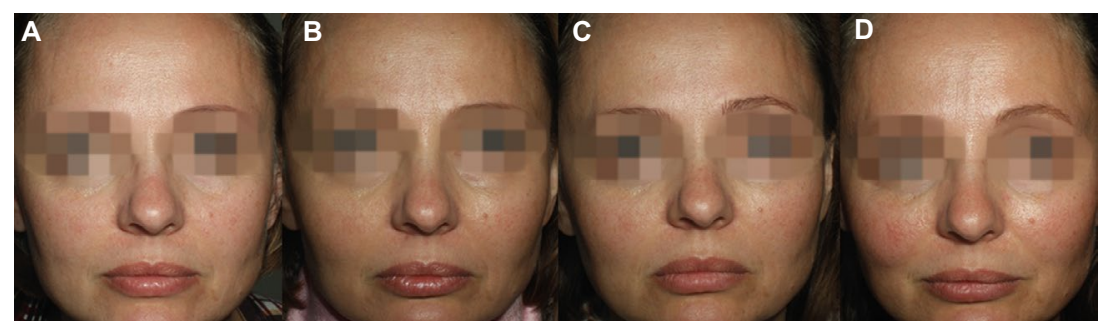

Figure 2 A 42-year-old subject who received CPM-26 on the left cheek and VYC-20 on the right cheek.

Notes: (A) Baseline, (B) M3 visit, (C) additional evaluation visit before touch-up and (D) MI2 visit after touch-up. MAS scores by the site-blinded evaluator were as follows: SCR - "2" on both sides; D0 - "0" on both sides; MI, M3 and M6 - "0" on CPM-26 side and "I" on VYC-20 side; and MI2 - "0" on both sides.

Abbreviations: CPM-26, Cohesive Polydensified Matrix ${ }^{\circledR} 26$ mg/mL HA gel; VYC-20, Vycross ${ }^{\circledR} 20$ mg/ml HA gel; M3, month 3; MI2, month I2; MAS, Merz Aesthetics Scale; SCR, screening; D0, day 0; MI, month I; M6, month 6; HA, hyaluronic acid. 

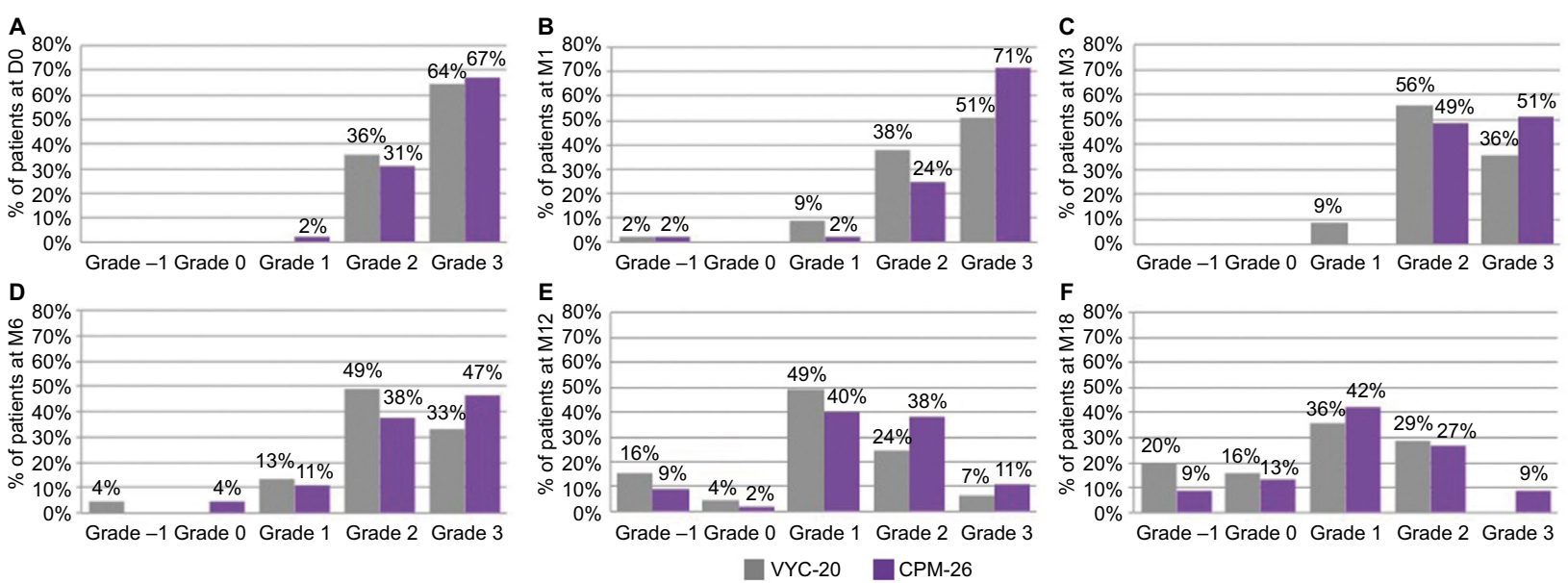

Figure 3 Frequency charts of GAIS grades as assessed by site-blinded evaluator at (A) D0, (B) MI, (C) M3, (D) M6, (E) MI2 and (F) MI8.

Note: CPM-26 is shown in purple and VYC-20 in gray.

Abbreviations: GAIS, Global Aesthetic Improvement Scale; D0, day 0; MI, month I; M3, month 3; M6, month 6; MI2, month 12; MI8, month 18; CPM-26, Cohesive Polydensified Matrix ${ }^{\circledR} 26 \mathrm{mg} / \mathrm{mL}$ HA gel; VYC-20, Vycross ${ }^{\circledR} 20 \mathrm{mg} / \mathrm{ml}$ HA gel; HA, hyaluronic acid.

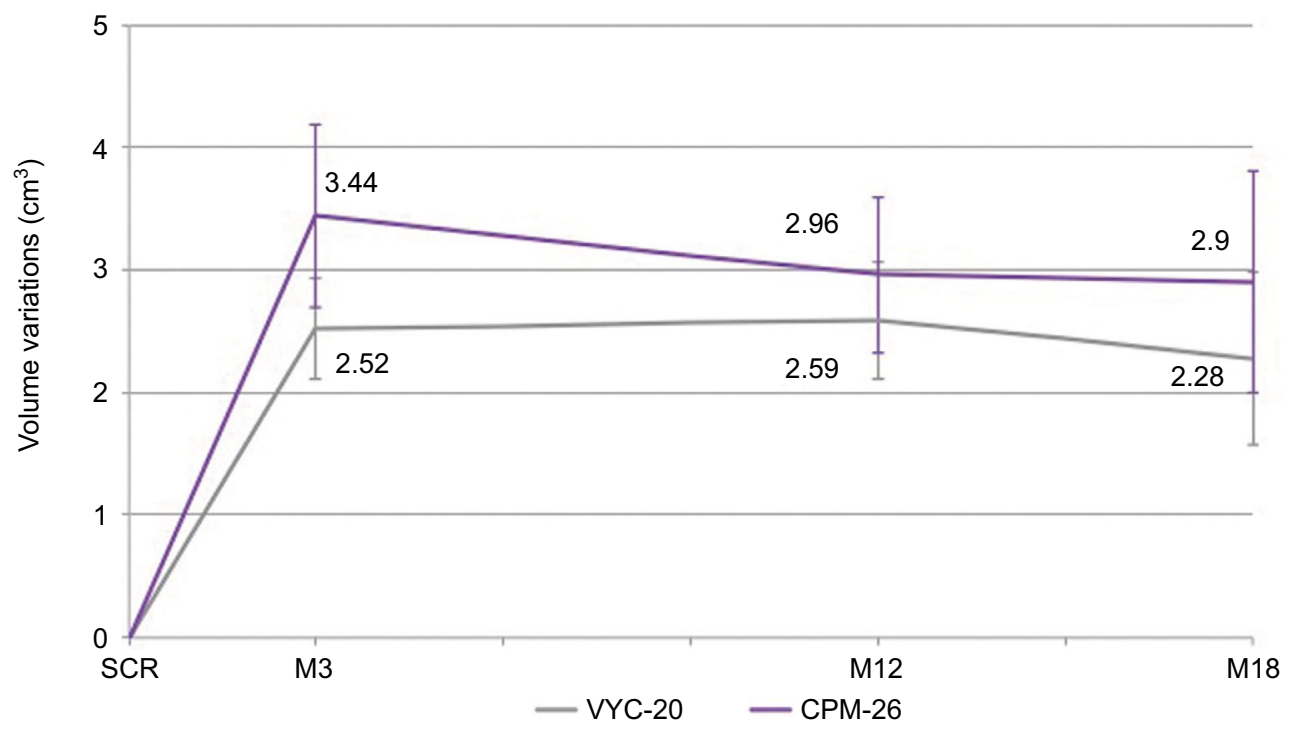

Figure 4 Anatomical volume variations with standard deviations, significant at $M 3$ (two-sided Wilcoxon signed-rank test; $p=0.0003$ ).

Abbreviations: M3, month 3; SCR, screening; MI2, month I2; MI8, month I8; VYC-20, Vycross ${ }^{\circledR} 20$ mg/ml HA gel; CPM-26, Cohesive Polydensified Matrix ${ }^{\circledR} 26$ mg/mL $\mathrm{HA}$ gel; HA, hyaluronic acid.

side than on the VYC-20 side at M1, M3, M12 and M18 visits $(p=0.0032, p=0.0074, p=0.0384$ and $p=0.0110$, respectively).

The results of the objective anatomical volume variations evaluation (LifeViz ${ }^{\mathrm{TM}}$ ) from baseline to M3, M12 and M18 are shown in Figure 4. CPM-26 showed visibly more volume augmentation at all time points, and the difference was significant at M3 $\left(3.44 \pm 1.49\right.$ vs $2.52 \pm 0.83 \mathrm{~cm}^{3}$; CPM-26 vs VYC-20; $\mathrm{n}=30 ; p=0.0003)$. Sustained volume augmentation was further detected at M12 (2.96 \pm 1.28 vs 2.59 \pm 0.96 ; $\mathrm{n}=26 ; p=0.0815)$ and M18 (2.90 \pm 1.80 vs $2.28 \pm 1.41 ; \mathrm{n}=26$; $p=0.0967$ ) for both products. Examples of subjects with the corresponding 3D images in pseudocolors are shown in Figures 5-7.

Both products showed excellent safety results. Transient injection-site reactions reported in this study were of mildto-moderate intensity.

\section{Discussion}

To our knowledge, this was the first controlled, randomized clinical study comparing two volumizing HA dermal fillers in a split-face design.

The results obtained from the site-blinded evaluator's rating based on MAS correspond to the expected level of 


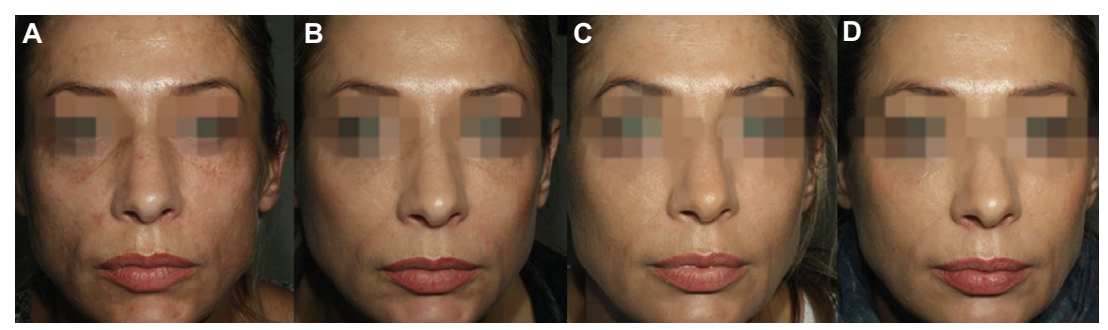

Figure 5 A 42-year old subject with CPM-26 on the right cheek and VYC-20 on the left cheek.

Notes: (A) SCR, (B) M3, (C) MI2 and (D) MI8 visits. GAIS grades on both sides: at M3, grade 3; at MI2, grade 2 and at MI8, grade 2; MAS grades on both sides: at SCR, grade 2; at $M 3$, grade 0 and at $M 18$, grade $I$.

Abbreviations: CPM-26, Cohesive Polydensified Matrix ${ }^{\circledR} 26$ mg/mL HA gel; VYC-20, Vycross ${ }^{\circledR} 20$ mg/ml HA gel; SCR, screening; M3, month 3; MI2, month I2; MI8, month 18; GAIS, Global Aesthetic Improvement Scale; MAS, Merz Aesthetics Scale; HA, hyaluronic acid.
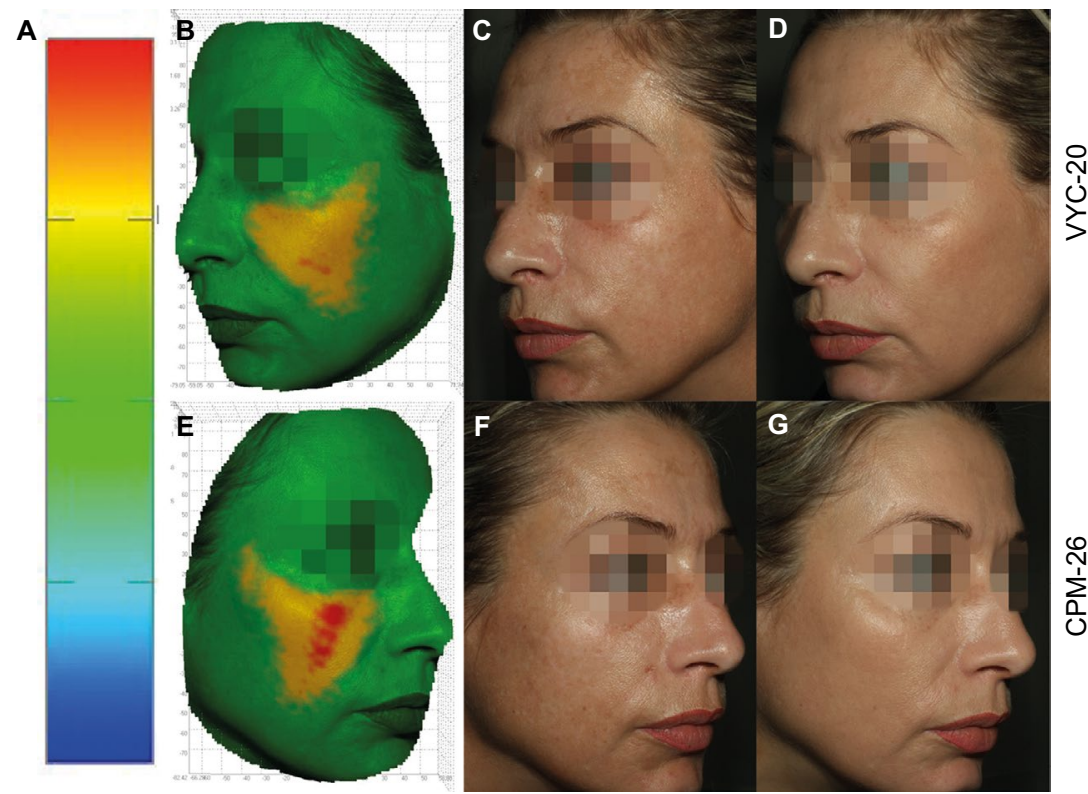

Figure 6 A 42-year old subject with CPM-26 on the right cheek and VYC-20 on the left cheek.

Notes: (A) Scale represents distance in millimeters for any given point between the pre- and postinjection images' scale. (B and E) Pseudocolor image of volume change (M3 vs SCR). (C and F) SCR photos. (D and G) M3 photos. Volume gain in cubic centimeters is shown in yellow-red; volume loss is shown in blue. Green color indicates that the matching of the zone is done with negligible volume difference.

Abbreviations: CPM-26, Cohesive Polydensified Matrix ${ }^{\circledR} 26$ mg/mL HA gel; VYC-20, Vycross ${ }^{\circledR} 20$ mg/ml HA gel; M3, month 3; SCR, screening; HA, hyaluronic acid.

performance of the products. Best grades were observed at M1 and M3, and non-inferiority of CPM-26 to VYC-20 was demonstrated at M3. These results were corroborated by the GAIS rating, which showed that at M1, a total of $71 \%(n=32)$ and $51 \%(n=23)$ of subjects were very much improved on the CPM-26 and VYC-20 sides, respectively. Significantly more subjects showed best grades in terms of esthetic improvement on the CPM-26 side than on the VYC-20 side at the $\mathrm{M} 1$ and $\mathrm{M} 3$ visits.

From M6 to M18, a general decrease in facial volume rated on MAS on both sides was supported by the decrease in GAIS scores. This can be explained by the beginning of gradual degradation of the implants by the endogenous hyaluronidases, which was also reported by Micheels et al ${ }^{9}$ at the same threshold. ${ }^{9}$
Nevertheless, the long-term duration of the volumizing effect was maintained up to M18 with 54\% $(\mathrm{n}=22)$ and 51\% $(n=19)$ of MAS scores improved by at least 1 grade on CPM26 and VYC-20 sides, respectively. On the GAIS, a total of $78 \%$ and $65 \%$ of scores were at least improved (subjects with grades 1, 2 and 3) on CPM-26 and VYC-20 sides, respectively. The investigators recommend an additional injection in $\sim 18$ months with a smaller quantity of the injected product in order to maintain the desired volumizing effect.

As the esthetics scales remain a relatively subjective tool, the authors sought an objective technique to quantify the amplitude of the esthetic effect. At present, such methods remain exploratory and their use in clinical trials is not very frequent. ${ }^{7}$ Magnetic resonance imaging (MRI) permits an accurate soft-tissue discrimination when used for evaluation 

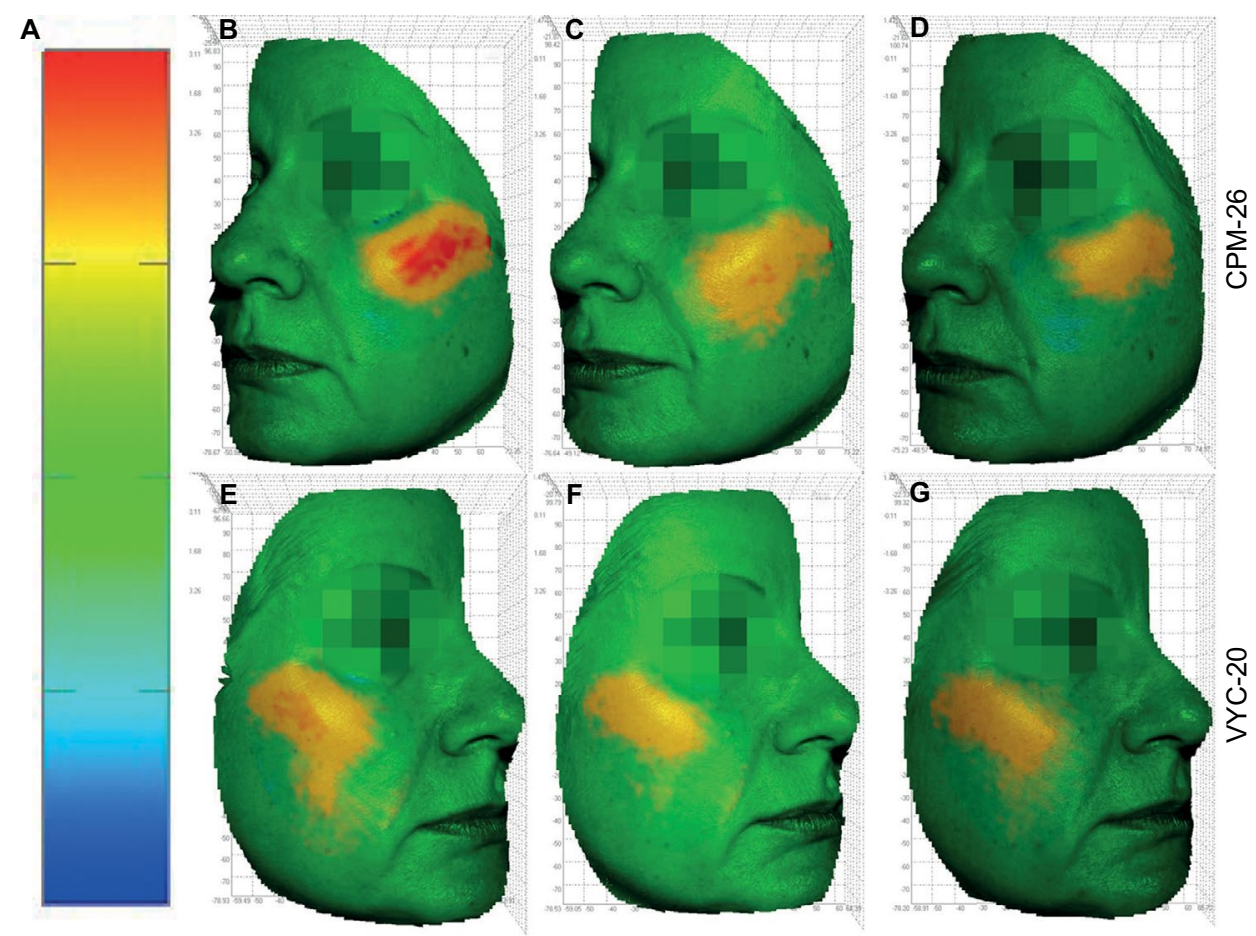

Figure 7 A 52-year old subject with VYC-20 on the right cheek and CPM-26 on the left cheek.

Notes: (A) Scale represents distance in millimeters for any given point between the pre- and postinjection images' scale. (B and E) M3 vs SCR. (C and F) MI2 vs SCR. (D and G) MI8 vs SCR. Volume gain in cubic centimeters is shown in yellow-red; volume loss is shown in blue. Green color indicates that the matching of the zone is achieved with negligible volume difference.

Abbreviations: VYC-20, Vycross ${ }^{\circledR} 20$ mg/ml HA gel; CPM-26, Cohesive Polydensified Matrix ${ }^{\circledR} 26$ mg/mL HA gel; M3, month 3; SCR, screening; MI2, month I2; MI8, month I8; HA, hyaluronic acid.

of dermal fillers. ${ }^{16,17}$ However, it remains an expensive and not easily accessible tool. Measurements of anatomical volume variations from 3D images represent another method reported in the literature ${ }^{13,18}$ and were chosen for the current study. This allowed relative comparison between right and left upper cheeks previously injected with different products. Even though it was performed in a limited number of subjects, a significant difference in terms of volume augmentation was shown in favor of CPM-26 at M3.

The fact that both products created a larger volume at the posttreatment visits than originally injected could be a result of the hygroscopic properties of the gels. Short-term volume increase may be due to HA's propensity to expand after implantation. Its ability to degrade in an isovolumetric manner, i.e., to maintain volume as it is degraded by attracting water molecules, ${ }^{19}$ contributes to the long-lasting results observed at M18.

In a study by Becker et al, ${ }^{16}$ which used MRI to detect volume augmentation created with CPM-26, a significantly higher amount of HA was present in vivo at M1 than the injected volume. In fact, the authors observed accumulation of HA and bound water in deeper compartments and the areas were associated with an increase in tissue vascularization and thickness. Measurements of volume variations on the photographs do not allow visualization of the subcutaneous structures, so our findings are complemented by the observations made by Becker et al.

Regarding the four subjects who received touch-ups with VYC-20 for correction of posttreatment asymmetry, it is difficult to explain the reasons for this observation, given that the initial treatment was performed with the same filler quantity and injection technique on both sides. The investigators agreed that this phenomenon should be interpreted as a failure in the performance of the product, caused by either low volumizing capacity or product migration.

HA filler properties and their associated clinical outcomes can be linked to several parameters such as HA concentration and manufacturing technology, among others. The HA concentration influences the gel consistency of the final product. With the same amount of crosslinks, a higher concentration of HA will result in a thicker gel $^{20}$ that is more resistant to dynamic forces and more suitable for deeper injection, thus potentially explaining the higher volumizing capacity of CPM-26 in the current study. However, this is not the only parameter to consider. Research has demonstrated that manufacturing technologies have an impact on matrix structure ${ }^{21,22}$ as well as gel rheological properties. ${ }^{23,24}$ CPM-26 is manufactured with CPM technology, which 
creates a product with variable cross-linking densities within the gel, where denser areas ensure a volumizing effect and less dense areas ensure a lower extrusion force, high cohesivity and tissue integration of the matrix. In contrast, VYC-20 is manufactured with Vycross technology in which there is a higher proportion of low-to-high molecular weight HA, allowing more crosslinking between the HA molecules. As a result, CPM-26 demonstrates a similar G prime to VYC-20 but has a higher resistance to dynamic compression (E prime). ${ }^{24}$ Both products have volumizing indications, but the clinical data from the current study suggest that a combination of manufacturing technology and high HA concentration may result in the highest volumizing capacity in vivo.

The current study was affected by the following limitations. First of all, MAS was designed for live evaluations, and volume assessments performed using printed photographs were found to be a challenging task. The primary end point was based on the photographic rating by the independent evaluator blinded to visit chronology. She rated approximately half of the subjects as having full upper cheeks (MAS grade 0) at baseline, in contrast to the blinded evaluator at the site who rated all subjects with MAS grades 2 and 3 at a live assessment. Consequently, based on the photographic assessment, these subjects could not improve any further. This could explain the fact that the responder rate on photographic evaluation was lower than the responder rate on live assessment in the current study and other published clinical data. ${ }^{9,13,25,26}$

However, as both fillers were equally affected by these methodological limitations, a comparison between the two was still possible. Therefore, the demonstrated non-inferiority combined with numerical superiority of CPM-26 to VYC-20 at $\mathrm{M} 3$ remains a valid outcome. For the assessment of the absolute performance of the products, the authors have reorientated the study objective toward the live rating on MAS, which yielded an expected responder rate for both products and also confirmed non-inferiority of CPM-26 versus VYC20 at M3. This experience should provide guidance for the design of future clinical studies.

Another study limitation concerns the fixed maximum volume $(2 \mathrm{~mL})$ of fillers restricted to a single injection session. It may not have been sufficient for optimal correction in certain subjects with severe facial volume loss. In fact, in the recently published studies on VYC-20, subjects were exposed to higher volumes of the product injected at different sites over several touch-up sessions. The authors believe that for this reason, the published rates of volume augmentation and global esthetic improvement were higher than in the current study. It should also be noted that the rating scales and the effectiveness end points used in these studies were not exactly the same. ${ }^{13,25,26}$

\section{Conclusion}

The primary objective was achieved with the demonstration of the non-inferiority combined with numerical superiority of CPM-26 versus VYC-20 at M3 based on MAS.

Significantly more subjects showed better esthetic improvement on the GAIS scale on the CPM-26 side than on the VYC-20 side at M1, M3, M12 and M18 visits. Analysis of anatomical volume variations based on $3 \mathrm{D}$ images showed that a larger volume was measured at all time points compared to the volume of the injected products at D0. CPM-26 demonstrated a significantly stronger volumizing capacity than VYC-20 at M3.

The results of MAS, GAIS and 3D anatomical volume variations collectively confirm that for both products, optimal correction was achieved at M3 and the long-lasting volumizing effect was maintained up to M18. CPM-26 demonstrated a favorable effectiveness profile for midfacial volume enhancement.

\section{Acknowledgments}

The authors would like to thank Alexandra Faure from Anteis S.A. for the support in the realization of this study and Anne Kunz from SCIderm $\mathrm{GmbH}$ for the statistical analysis of the results. This clinical study (study number MOD-1201) was sponsored by Anteis S.A., Geneva, Switzerland (a wholly owned subsidiary of Merz Pharmaceuticals GmbH, Frankfurt am Main, Germany) and was conducted at the SCIderm GmbH (Hamburg, Germany) investigational site.

\section{Disclosure}

M Kerscher has received research support and has conducted clinical trials for Merz Pharmaceuticals $\mathrm{GmbH}$ and has acted as a speaker and/or investigator for Merz, Kythera, Q-Med/Galderma and Pierre Fabre. K Agsten has received research support and has conducted clinical trials for Merz Pharmaceuticals GmbH. M Kravtsov was an employee of Anteis S.A. W Prager has received research support and has conducted clinical trials for Merz Pharmaceuticals $\mathrm{GmbH}$ and has acted as a speaker and/or investigator for Merz, Q-Med/Galderma, Allergan, Beiersdorf, SkinCeuticals, Cynosure and Lumenis. The authors report no other conflicts of interest in this work. 


\section{References}

1. Coleman SR, Grover R. The anatomy of the aging face: volume loss and changes in 3-dimensional topography. Aesthet Surg J. 2006;26(1S):S4-S9.

2. Raspaldo H, Gassia V, Niforos FR, Michaud T. Global, 3-dimensional approach to natural rejuvenation: part 1 - recommendations for volume restoration and the periocular area. J Cosmet Dermatol. 2012;11(4):279-289

3. Wollina U. Facial rejuvenation starts in the midface: three-dimensional volumetric facial rejuvenation has beneficial effects on nontreated neighboring esthetic units. J Cosmet Dermatol. 2016;15(1):82-88.

4. Wollina U. Midfacial rejuvenation by hyaluronic acid fillers and subcutaneous adipose tissue - a new concept. Med Hypotheses. 2015;84(4):327-330.

5. Philipp-Dormston WG, Eccleston D, De Boulle K, et al. A prospective, observational study of the volumizing effect of open-label aesthetic use of Juvéderm ${ }^{\circledR}$ VOLUMA ${ }^{\circledR}$ with lidocaine in mid-face area. J Cosmet Laser Ther. 2014;16:171-179.

6. Ascher B, Coleman S, Alster T, et al. Full scope of effect of facial lipoatrophy: a framework of disease understanding. Dermatol Surg. 2006;32(8):1058-1069.

7. Goldman MP, Skover GR, Payonk GS. Three-dimensional imaging techniques in the assessment of facial volume augmentation. J Drugs Dermatol. 2009;8(12):1113-1119.

8. Mills DC, Camp S, Mosser S, Sayeg A, Hurwitz D, Ronel D. Malar augmentation with a polymethylmethacrylate-enhanced filler: assessment of a 12-month open-label pilot study. Aesthet Surg J. 2013;33(3):421-430.

9. Micheels P, Vandeputte J, Kravtsov M. Treatment of age-related midface atrophy by injection of cohesive polydensified matrix hyaluronic acid volumizer. J Clin Aesthet Dermatol. 2015;8(3):28-34.

10. Little JW. Volumetric perceptions in midfacial aging with altered priorities for rejuvenation. Plast Reconstr Surg. 2000;105(1):252-266.

11. Hoffmann K; Juvederm Voluma Study Investigators Group. Volumizing effects of a smooth, highly cohesive, viscous $20-\mathrm{mg} / \mathrm{mL}$ hyaluronic acid volumizing filler: prospective European study. BMC Dermatol. 2009;9:9.

12. Raspaldo H. Volumizing effect of a new hyaluronic acid sub-dermal facial filler: a retrospective analysis based on 102 cases. J Cosmet Laser Ther. 2008;10(3):134-142.

13. Jones D, Murphy DK. Volumizing hyaluronic acid filler for midface volume deficit: 2-year results from a pivotal single-blind randomized controlled study. Dermatol Surg. 2013;39(11):1602-1612.
14. Carruthers J, Flynn TC, Geister TL, et al. Validated assessment scales for the mid face. Dermatol Surg. 2012;38(2 Spec No.):320-332.

15. Narins RS, Brandt F, Leyden J, Lorenc ZP, Rubin M, Smith S. A randomized, double-blind, multicenter comparison of the efficacy and tolerability of Restylane versus Zyplast for the correction of nasolabial folds. Dermatol Surg. 2003;29(6):588-595.

16. Becker M, Balague N, Montet X, et al; LIPO and Metabolism Group. Hyaluronic acid filler in HIV-associated facial lipoatrophy: evaluation of tissue distribution and morphology with MRI. Dermatology. 2015;230(4):367-374.

17. Ginat DT, Schatz CJ. Imaging features of midface injectable fillers and associated complications. AJNR Am J Neuroradiol. 2013; 34(8):1488-1495.

18. Kestemont $\mathrm{P}$, Cartier H, Trevidic P, et al. Sustained efficacy and high patient satisfaction after cheek enhancement with a new hyaluronic acid dermal filler. J Drugs Dermatol. 2012;11(1 suppl):s9-s16.

19. Patel U, Fitzgerald R. Facial shaping: beyond lines and folds with fillers. J Drugs Dermatol. 2010;9(8 suppl ODAC Conf pt 2):s129-s137.

20. Ramos-E-Silva M, Fonteles LA, Lagalhard CS, Fucci-da-Costa AP. STYLAGE ${ }^{\circledR}$ : a range of hyaluronic acid dermal fillers containing mannitol. Physical properties and review of the literature. Clin Cosmet Investig Dermatol. 2013;6:257-261.

21. Flynn TC, Thompson DH, Hyun SH, Howell DJ. Ultrastructural analysis of 3 hyaluronic acid soft-tissue fillers using scanning electron microscopy. Dermatol Surg. 2015;41(suppl 1):S143-S152.

22. Mondon K, Dadras M, Tillier J, Gavard Molliard S. Influence of the macro- and/or microstructure of cross-linked hyaluronic acid hydrogels on the release of two model drugs. J Glycobiol. 2016;5:119.

23. Gavard Molliard S, Albert S, Mondon K. Key importance of compression properties in the biophysical characteristics of hyaluronic acid soft-tissue fillers. J Mech Behav Biomed Mater. 2016;61:290-298.

24. Stocks D, Sundaram H, Michaels J, Durrani MJ, Wortzman MS, Nelson DB. Rheological evaluation of the physical properties of hyaluronic acid dermal fillers. J Drugs Dermatol. 2011;10(9):974-980.

25. Callan P, Goodman GJ, Carlisle I, et al. Efficacy and safety of a hyaluronic acid filler in subjects treated for correction of midface volume deficiency: a 24 month study. Clin Cosmet Investig Dermatol. 2013;6:81-89.

26. Few J, Cox SE, Paradkar-Mitragotri D, Murphy DK. A multicenter, single-blind randomized, controlled study of a volumizing hyaluronic acid filler for midface volume deficit: patient-reported outcomes at 2 years. Aesthet Surg J. 2015;35(5):589-599.
Clinical, Cosmetic and Investigational Dermatology

\section{Publish your work in this journal}

Clinical, Cosmetic and Investigational Dermatology is an international, peer-reviewed, open access, online journal that focuses on the latest clinical and experimental research in all aspects of skin disease and cosmetic interventions. This journal is included on PubMed. The manuscript management system is completely online and includes a very quick and fair peer-review system, which is all easy to use. Visit http://www.dovepress.com/testimonials.php to read real quotes from published authors 